\title{
Stability and convergence analysis for a new phase field crystal model with a nonlocal Lagrange multiplier
}

\author{
Shimin Lin ${ }^{1}$, Junying $\mathrm{Cao}^{2}$, Jun Zhang ${ }^{3}$, and Tao Sun $^{4}$ \\ ${ }^{1}$ Jimei University \\ ${ }^{2}$ Guizhou Minzu University \\ ${ }^{3}$ Guizhou University of Finance and Economics \\ ${ }^{4}$ Shanghai Lixin University of Accounting and Finance
}

June 27, 2020

\begin{abstract}
In this work, an energy stable numerical scheme is proposed to solve the PFC model with a nonlocal Lagrange multiplier. The construction of the numerical scheme is based on invariant energy quadratization (IEQ) technique to transform a nonlinear system into a linear system, while the time variables is discretized by second order scheme. The stability term in the new scheme can balance the influence of nonlinear term. Moreover, we obtain the results of unconditional energy stability, uniqueness and uniform boundedness of numerical solution, and the numerical scheme is convergent with order of $\$ \backslash$ mathcal $\{\mathrm{O}\}\left(\backslash \operatorname{delta} \mathrm{t}^{\wedge} 2\right) \$$. Several numerical tests are conducted to confirm the theoretical results.
\end{abstract}

\section{Hosted file}

CAPFC2.pdf available at https://authorea.com/users/337367/articles/462959-stability-andconvergence-analysis-for-a-new-phase-field-crystal-model-with-a-nonlocal-lagrangemultiplier 
figures/CG1-0/CG1-0-eps-converted-to.pdf 
figures/CG1-600/CG1-600-eps-converted-to.pdf 
figures/CG1-100/CG1-100-eps-converted-to.pdf 
figures/CG1-1000/CG1-1000-eps-converted-to.pdf 
figures/CG1-300/CG1-300-eps-converted-to.pdf 
figures/CG1-2000/CG1-2000-eps-converted-to.pdf 
figures/CGL1-0/CGL1-0-eps-converted-to.pdf 
figures/CGL1-100/CGL1-100-eps-converted-to.pdf 
figures/CGL1-1000/CGL1-1000-eps-converted-to.pdf 
figures/CG1-ENG/CG1-ENG-eps-converted-to.pdf 
figures/CG1-ME/CG1-ME-eps-converted-to.pdf 
figures/CG3-0/CG3-0-eps-converted-to.pdf 
figures/CG3-600/CG3-600-eps-converted-to.pdf 
figures/CG3-100/CG3-100-eps-converted-to.pdf 
figures/CG3-1000/CG3-1000-eps-converted-to.pdf 
figures/CG3-300/CG3-300-eps-converted-to.pdf 
figures/CG3-2000/CG3-2000-eps-converted-to.pdf 
figures/CGL3-0/CGL3-0-eps-converted-to.pdf 
figures/CGL3-300/CGL3-300-eps-converted-to.pdf 
figures/CGL3-1000/CGL3-1000-eps-converted-to.pdf 
figures/CG3-ENG/CG3-ENG-eps-converted-to.pdf 
figures/CG3-ME/CG3-ME-eps-converted-to.pdf 
figures/PSB1-0/PSB1-0-eps-converted-to.pdf 
figures/PSB1-30/PSB1-30-eps-converted-to.pdf 
figures/PSB1-10/PSB1-10-eps-converted-to.pdf 
figures/PSB1-40/PSB1-40-eps-converted-to.pdf 
figures/PSB1-20/PSB1-20-eps-converted-to.pdf 
figures/PSB1-1000/PSB1-1000-eps-converted-to.pdf 
figures/PSB1-ENG/PSB1-ENG-eps-converted-to.pdf 
figures/PSB1-ME/PSB1-ME-eps-converted-to.pdf 
figures/PSB2-0/PSB2-0-eps-converted-to.pdf 
figures/PSB2-10/PSB2-10-eps-converted-to.pdf 
figures/PSB2-40/PSB2-40-eps-converted-to.pdf 
figures/PSB2-30/PSB2-30-eps-converted-to.pdf 
figures/PSB2-20/PSB2-20-eps-converted-to.pdf 
figures/PSB2-1000/PSB2-1000-eps-converted-to.pdf 
figures/PSB2-ENG/PSB2-ENG-eps-converted-to.pdf 
figures/PSB2-ME/PSB2-ME-eps-converted-to.pdf 